\title{
Fatigue Assessment of Brazed T-Joints Based on Damage Tolerance Including Residual Stress Effects
}

\begin{abstract}
The fatigue crack growth behavior of a brazed joint is characterized by a Paris-exponent that is much higher than the one of bulk metals, which means that brazed components have only a short residual fatigue life after the initiation of a fatigue crack. Therefore, the threshold of fatigue crack growth of brazed joints is of particular importance in the fatigue analysis of brazed components and needs to be understood well. Residual stresses have to be considered in crack growth analysis in the threshold regime. The corresponding effects are explored experimentally and theoretically. The stress intensity factors due to the residual stresses in a brazed T-joint of compressor impellers were measured by using the cut compliance method. It was found that residual stresses are present but relatively small. They do not affect the endurance stress significantly. A concept for an endurance analysis of a brazed T-joint that includes imperfections and residual stresses is suggested.
\end{abstract}

KEYWORDS: fatigue crack, endurance limit, residual stress, stress intensity factor, threshold, brazed joint, notch, T-joint

\section{Introduction}

In brazed joints pores and zones of reduced bonding strength are usually inevitable. Therefore, highly loaded brazed components such as compressor impellers need to be designed according to the principles of damage tolerance, which include a defect assessment based on fracture mechanics [1,2]. Under mechanical loading, complex triaxial stresses form in the thin brazing zone due to the different elastic-plastic properties of the filler metal and the base material and the constraining effect of the base material. As a result, the ultimate tensile strength of the bond can be several times higher than the strength of the unconstrained layer material $[3,4]$. This effect also influences the fatigue behavior, namely, the endurance limit of brazed components.

The characteristic feature of fatigue crack growth in brazed joints is its extremely steep slope of the $d a / d N$-curve, which manifests in a very high exponent in a power law representation of the crack growth rate $d a / d N$ as a function of $\Delta K$ ("Paris' law") $[5,6]$. This means that a fatigue crack-once it is startedwill accelerate rapidly and lead to a fracture. Thus, to guarantee safety with respect to fatigue, all cyclic load components, even those with relatively few numbers of cycles like the on/off load cycles, should stay well below the threshold of fatigue crack growth. However, little is known about the threshold behavior of brazed joints.

In the threshold regime of fatigue crack growth, the effect of residual stresses often plays an important role and should be considered principally in fatigue assessment. Little is known about residual stresses in brazed joints and their effects on fatigue. Gross residual stresses are rather unlikely to occur since the brazing process acts like a stress relief heat treatment. However, local residual stresses are likely to be generated during the final cooling due to the differences in thermal expansion between base and filler material and may affect the threshold behavior. In the present investigation they are measured and taken into account in the fatigue assessment. In order to perform a fatigue analysis based on fracture mechanics, particularly the stress intensity factor (SIF) caused by the residual stresses is required.

An example of a compressor impeller is shown in Fig. 1. The base material is martensitic stainless steel X3CrNiMo13-4 and the filler metal Au-18Ni. Brazing is used to connect the top plate of the impeller

\footnotetext{
Manuscript received May 14, 2009; accepted for publication December 11, 2009; published online February 2010.

${ }^{1}$ Mat-Tec AG, Winterthur, Switzerland.

${ }^{2}$ EMPA - Swiss Federal Laboratories for Materials Testing and Research, Dübendorf, Switzerland.
} 


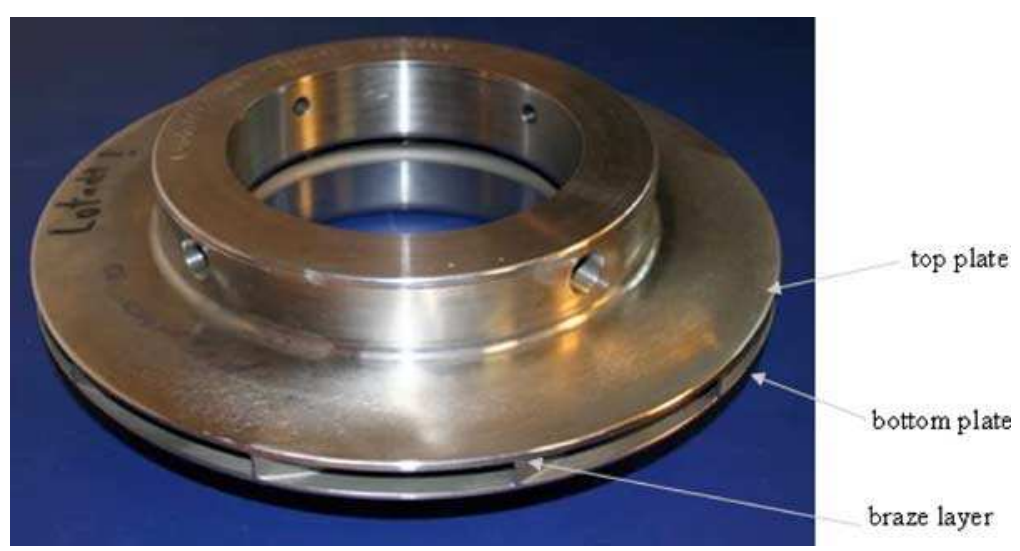

FIG. 1-Example of a compressor impeller.

to the blades, which are part of the bottom plate in Fig. 1. Thus, the brazed joints are typically T-shaped, so there is a global stress concentration at the edge of the braze seam.

In a fatigue analysis of a brazed component such as the impeller, the special features mentioned above-including global stress concentration, local defects, steep $d a / d N$-curves, and residual stressesshould be taken into account adequately. The aim of the present investigation was to explore a practical way of how to deal with these issues. In this paper, the fatigue behavior is reviewed, and the importance of the threshold of fatigue crack growth in endurance is emphasized. Near the threshold, residual stresses often play an important role in fatigue crack growth. It is shown how they can be measured and assessed. For the measurement the cut compliance (CC) method was adapted. In the present paper it is shown how the CC-method was adapted to measure the residual stress effects in an impeller. Furthermore a practical defect assessment procedure of a brazed T-joint is suggested.

\section{Fatigue Behavior of Brazed Joints}

The fatigue crack growth rates of brazed joints of X3CrNiMo13-4 steel with Au-18Ni filler material (as applied in the case of the compressor impeller shown in Fig. 1) were measured on double cantilever beam (DCB) specimens. The experimental data in terms of crack growth rate, $d a / d N$, as a function of $\Delta K$ (" $d a / d N$-curve") for several stress ratios are reported in Refs 5 and 6. Some $d a / d N$-curves are shown in Fig. 2. The parameters $C, n$, and $\Delta K_{\text {th }}$ that correspond to an approximation of the $d a / d N$-curve by

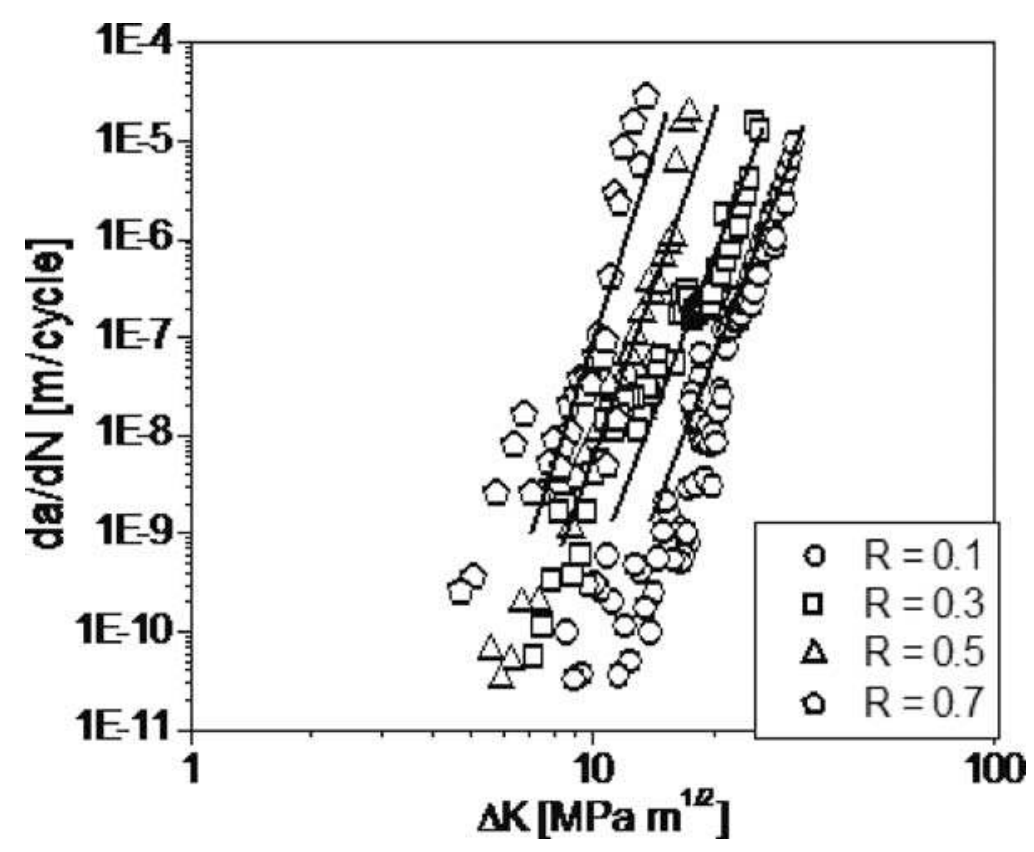

FIG. 2-Fatigue crack propagation (da/dN- $\Delta K)$ curves for $R=0.1,0.3,0.5$, and 0.7 (from Ref 6). 
TABLE 1 -Threshold values $\Delta K_{\text {th }}$ and calculated $C$ and $n$ parameters at different $R$ values [6].

\begin{tabular}{lccc}
\hline$R$ & $\Delta K_{\mathrm{th}}\left(\mathrm{MPa} \cdot \mathrm{m}^{0.5}\right)$ & $C$ & $n$ \\
\hline 0.1 & 9 & $1.309 \times 10^{-22}$ & 11.17 \\
0.3 & 7 & $4.071 \times 10^{-23}$ & 12.17 \\
0.5 & 6 & $7.234 \times 10^{-22}$ & 12.64 \\
0.7 & 4 & $8.489 \times 10^{-21}$ & 12.81 \\
\hline
\end{tabular}

$$
\begin{gathered}
\frac{d a}{d N}=C \cdot \log \Delta K_{I}^{n}, \quad \text { for } \Delta K_{I}>\Delta K_{\mathrm{th}} \\
\frac{d a}{d N}=0, \quad \text { for } \Delta K_{I}<\Delta K_{\mathrm{th}}
\end{gathered}
$$

are given in Table 1 for some $R$-ratios. $R$ denotes the stress ratio

$$
R=K_{\min } / K_{\max }
$$

with $K_{\min }$ and $K_{\max }$ being the minimum and maximum SIF, respectively, of a load cycle.

Compared with the fatigue crack growth rate in bulk metals, where the Paris-exponent $n$ is typically in the range of $2.5<n<4$, the values of $n$ shown in the Table 1 are extremely high. This unusually high slope of the $d a / d N$-curves can be explained by the fact that the range of $\Delta K_{I}$, in which fatigue crack growth is principally possible, i.e.

$$
\Delta K_{\mathrm{th}}(R)<\Delta K<(1-R) \cdot K_{\mathrm{cf}}
$$

is relatively narrow since the threshold value $\Delta K_{\text {th }}$ of the brazing is-as discussed in the Fatigue Crack Growth Threshold section — of a similar level as the one of bulk steel, whereas the upper bound $K_{\mathrm{cf}}$ (which denotes the critical SIF in cyclic loading) is much lower than the corresponding value of bulk steel. Simply, the $d a / d N$-curve has to be much steeper than the one of bulk material to fit in the narrow range of $\Delta K$ as given in Eq 3, which is less than one decade wide. Or in physical terms: The mechanisms that cause acceleration in the upper range and the ones that lead to retardation in threshold regime of the $d a / d N$-curve interfere with the Paris-behavior nearly in the entire range of $\Delta K$.

From a practical point of view the high $n$-values mean that crack growth accelerates rapidly as soon as the crack starts to grow. This behavior leaves only little space for a safe-life-design of a brazed component. Taking into account the relatively high natural scatter of threshold data on one hand and the uncertainties to calculate the applied stress range $\Delta K$ on the other, a defect-tolerant design based on a safe-life analysis appears to be too risky to be applied. Instead, it is appropriate to require endurance (i.e., infinite fatigue life) even for load cases that are applied in relatively small numbers, say, a few thousand times, like, e.g., the on-off load cycles of an impeller. In terms of fracture mechanics, endurance requires that no fatigue crack growth occurs for the crucial initial defect size $a_{0}$, i.e., that

$$
\Delta K\left(a_{0}\right)<\Delta K_{\mathrm{th}}(R)
$$

In the following we take a closer look on both sides of Eq 4 for a brazed T-joint.

\section{Stress Intensity Factor for a Crack in a T-Joint}

As a representative simple plane geometry, we consider a T-shaped structural part of unit thickness loaded by a bending moment $M$ and a normal tensile force $N$ per unit thickness (Fig. 3). At the stress concentra-

tion in the corner, a crack of depth $a$ is assumed to be present. Figure 4 shows the non-dimensional SIF $k_{\mathrm{Ib}}$ and $k_{\mathrm{It}}$, which are defined as

$$
k_{\mathrm{It}}=\frac{K_{I} \cdot W^{0.5}}{N} ; \quad k_{\mathrm{Ib}}=\frac{K_{I} \cdot W^{1.5}}{M}
$$

where the SIF $K_{I}$ was calculated numerically by a boundary element analysis.

As it is typical for a crack emanating from a stress concentration, there is a steep rise of $K_{I}(a)$ with increasing crack length $a$ in the short crack range, followed by a flatter part for larger crack depths $[7,8]$. 


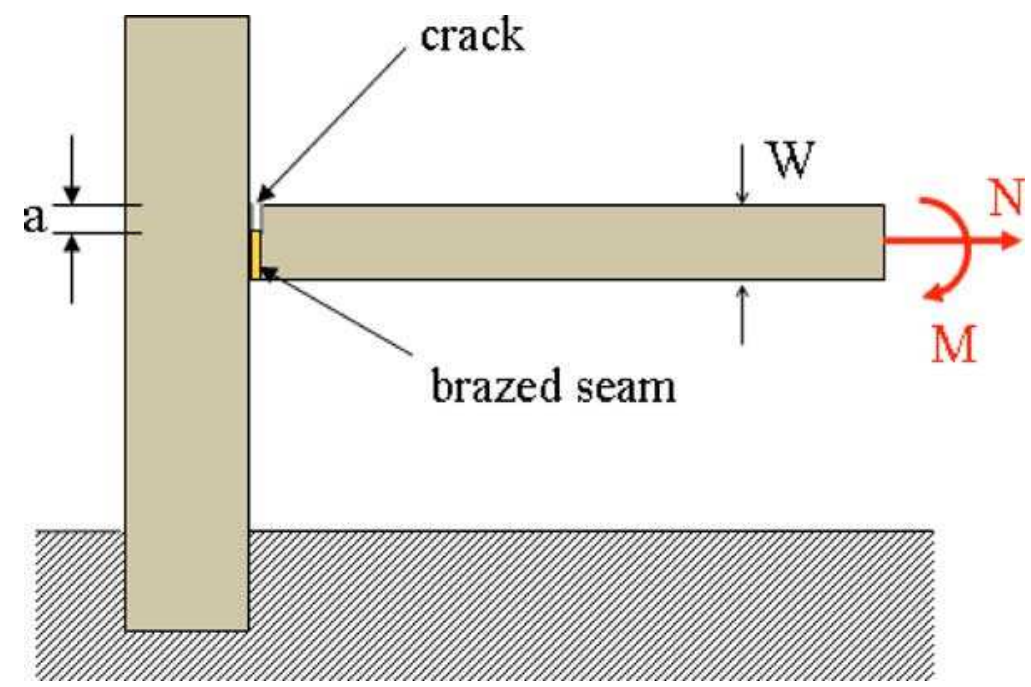

FIG. 3-T-shaped brazed joint with crack of depth a loaded by a bending moment $M$ and a normal force $N$.

Cracks in sharp corners like the one in a T-joint are difficult to be identified or measured by common methods of non-destructive testing, so their length is in general not known exactly. On the other hand-as it can be seen from Fig. 4 - little differences in crack depth cause large differences in $K_{I}$ in the initial state of crack growth. Therefore it is suggested in Refs 7 and 8 to cover the initial state of crack growth by extrapolating the subsequent flat part of $K_{I}(a)$ to $a=0$. This is achieved by fitting the $K_{I}(a)$-curve to the form

$$
K_{I}(a)=1.12 \cdot \sigma_{N} \cdot \sqrt{\pi\left(a+a_{N}\right)} \cdot Y(a / W)
$$

where:

$\sigma_{N}$ and $a_{N}=$ fitting constants and

$Y(a / W)=$ character of the curve in the range of deeper cracks.

Equation 6 represents the upper bound of $K_{I}$ for a short crack emanating from a notch or hot-spot of arbitrary shape regardless of the geometrical details of the corner such as its radius. For $a=0$ approximation 6 delivers a theoretical SIF of

$$
K_{I}(a=0)=1.12 \cdot \sigma_{N} \cdot \sqrt{\pi \cdot a_{N}}=: K_{I N}
$$

where:

the sign =: means "defined as."

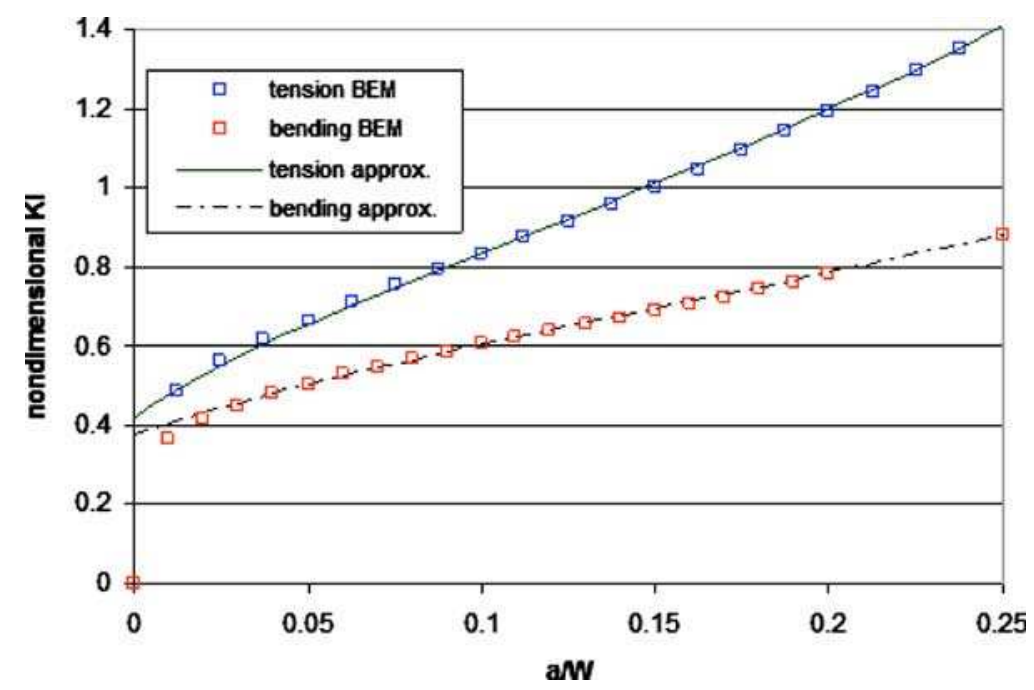

FIG. 4-Non-dimensional SIF $k_{I t}$ and $k_{I b}$ as defined in Eq 5 for the system shown in Fig. 2. 
$K_{I N}$ is called the notch SIF (NSIF). It can be interpreted as the "equivalent SIF" of a notch regardless of whether or not a real crack-like defect is present. Physically, a finite $K_{I}$ at $a=0$ cannot occur even at a very sharp notch, but it can occur in cases where a small crack-like defect disturbs the local geometry. However, $K_{I N}$ represents the worst case of $K_{I}$ of a short crack emanating from a sharp notch, including a small initial crack-like defect of unknown shape and depth.

Correspondingly, the endurance criterion 4 applied to a sharp notch or corner becomes

$$
\Delta K_{I N}<\Delta K_{\mathrm{th}}(R)
$$

where:

$\Delta K_{I N}=$ range of $K_{I N}$ in case of cyclic loading.

Criterion 8 is convenient for practical purposes since the initial crack size, which is poorly defined and difficult to be measured, does not appear explicitly in it. This concept has been successfully applied to predict the endurance limit of welded joints (Refs 7 and 8 and unpublished work).

In the present case of a T-joint as shown in Fig. 3, a curve fit in the sense of Eq 6 of the $K_{I}(a)$ data shown in Fig. 4 delivers

$$
K_{I}=\frac{2.293 \cdot N}{\sqrt{W}} \cdot \sqrt{\frac{a}{W}+0.0338} \cdot Y_{N}(a / W)
$$

with

$$
Y_{N}(a / W)=1-0.5454 \cdot\left(\frac{a}{W}\right)+5.8863 \cdot\left(\frac{a}{W}\right)^{2}-8.9417 \cdot\left(\frac{a}{W}\right)^{3}+15.809 \cdot\left(\frac{a}{W}\right)^{4}
$$

for a loading by a normal force $N$ and

$$
K_{I}=\frac{8.97 \cdot M}{W^{1.5}} \cdot \sqrt{\frac{a}{W}+0.0625} \cdot Y_{M}(a / W)
$$

with

$$
Y_{M}(a / W)=1-0.0298 \cdot\left(\frac{a}{W}\right)-0.2622 \cdot\left(\frac{a}{W}\right)^{2}+5.132 \cdot\left(\frac{a}{W}\right)^{3}-0.8777 \cdot\left(\frac{a}{W}\right)^{4}
$$

for a bending moment $M$. These fitting curves are also shown in Fig. 4. From Eqs 9a-9d, $K_{I N}$ as defined in Eq 7 turns out to be

$$
K_{I N}=\left(0.374 \cdot \sigma_{b}+0.422 \cdot \sigma_{t}\right) \cdot \sqrt{W}
$$

where:

$$
\sigma_{b}=\frac{6 M}{W^{2}} \quad \text { and } \quad \sigma_{t}=\frac{N}{W}
$$

denote the nominal bending and tensile stresses, respectively, that are introduced by $M$ and $N$ in the T-joint.

\section{Fatigue Crack Growth Threshold}

The threshold SIF $\Delta K_{\text {th }}$ of bulk metal is known to depend on the stress ratio $R$ approximately in the following way $[7,8]$ :

$$
\begin{gathered}
\Delta K_{\mathrm{th}}(R)=\Delta K_{\mathrm{th} 0} \cdot(1-R) \quad \text { for } R \leq 1-\frac{\Delta K_{\mathrm{th} / \mathrm{int}}}{\Delta K_{\mathrm{th} 0}} \\
\Delta K_{\mathrm{th}}=\Delta K_{\mathrm{th} / \mathrm{int}} \quad \text { for } R>1-\frac{\Delta K_{\mathrm{th} / \mathrm{int}}}{\Delta K_{\mathrm{th} 0}}
\end{gathered}
$$

where:

$\Delta K_{\mathrm{th} 0}=$ threshold SIF for $R=0$ and 


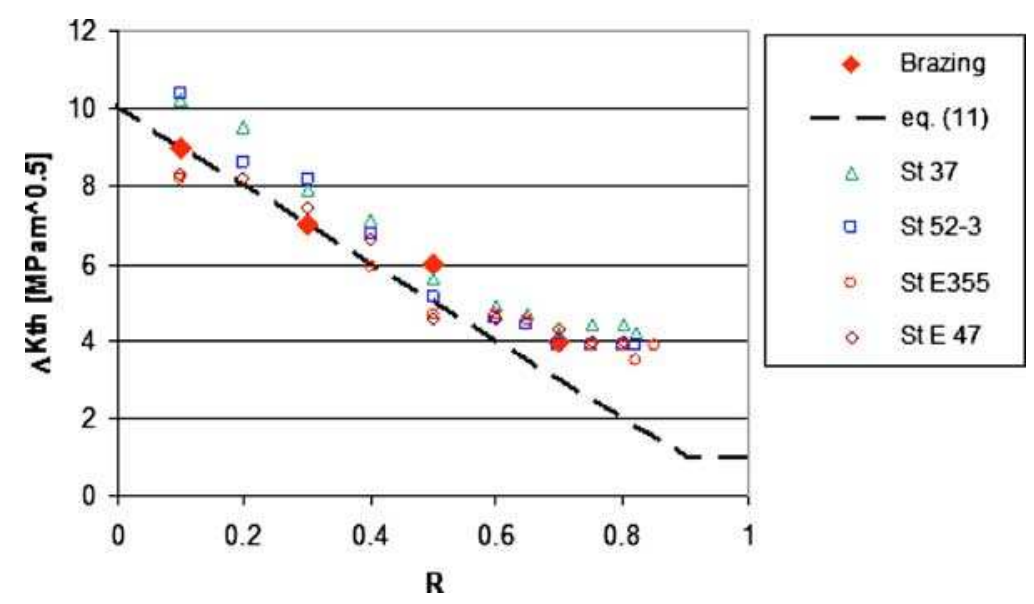

FIG. 5-Fatigue threshold of a brazed joint (Table 1) as a function of $R$, in comparison to data of structural steels from Ref 14 (cited in Ref 13).

$\Delta K_{\text {th/int }}=$ so-called intrinsic threshold.

The straight line described by Eq 11a can be physically explained by crack-closure effects that occur at relatively low $R$. $\Delta K_{\text {th/int }}$ forms a lower plateau that is reached for high $R$-values [9]. For bulk material the latter is known to be approximately proportional to Young's modulus $E$ [10-12]. It can be estimated by

$$
\Delta K_{\mathrm{th} / \mathrm{int}} \approx E \cdot d^{0.5}
$$

where:

$d=$ characteristic distance of about $d \approx 2.3 \times 10^{-10}(\mathrm{~m})$.

The general behavior of the threshold of brazed joints is expected to be more complex. In Fig. 5 the values from Table 1 are compared with the threshold values of some typical structural steels. They are roughly in line with the latter, which means that the threshold of brazed joints seems to be similar as the one of bulk steel.

At present time there are no experimental data for $R>0.7$ available, so it is unclear whether the curve of the $K_{\mathrm{th}}(R)$ flattens out as $R$ approaches 1 at the same plateau as bulk steel, or whether it further decreases to the plateau of bulk gold, which is obtained by Eq 12 with $E=78 \mathrm{MPa}$, to be about $\Delta K_{\mathrm{th} / \mathrm{int} / \mathrm{Au}}=1.1 \mathrm{MPa} \cdot \mathrm{m}^{0.5}$. From a theoretical point of view, the width of the braze layer is expected to play a certain role: If it is very narrow, then the threshold of bulk steel should apply. If it is wide enough to contain the active plastic zone caused by the cyclic load, the $\Delta K_{\text {th/int }}$ of bulk gold may apply. Anyway, in load cases of real components, the $R$-values are usually rather in the low and medium than in the very high range, so the question of the threshold at high $R$-values is not a very important one as far as practical applications are concerned. In the following, the dotted line shown in Fig. 5 is assumed to represent a lower bound, with $\Delta K_{\mathrm{th} / \mathrm{int} / \mathrm{Au}}$ denoting the lower plateau. The curve parameters corresponding to the dotted line in Fig. 5 are $\Delta K_{\mathrm{th} 0}=10 \mathrm{MPa} \cdot \mathrm{m}^{0.5}$ and $\Delta K_{\mathrm{th} / \mathrm{int}}=1.09 \mathrm{MPa} \cdot \mathrm{m}^{0.5}$.

\section{Residual Stress Effects on Crack Growth Threshold}

It is well known that the fatigue threshold is affected by residual stresses by their effect on the $R$-ratio. Often the residual stresses in the specimen, by which the threshold is determined, differ from the ones in the component. In cases like the present one, where $d a / d N$-curves are determined on DCB-specimens and applied to predict the behavior of a T-joint, the effect of the residual stresses on $\Delta K_{\mathrm{th}}(R)$ should be considered.

To include the residual stress effect in the analysis, it is suitable to represent the lower-bound curve shown in Fig. 5 in the plane $\Delta K$ versus $K_{\max }$, which is obtained by eliminating $R$ from Eqs 11 a and 11 by means of Eq 2. Therewith, the endurance criterion 4 becomes

$$
K_{\max } \leq \Delta K_{\mathrm{th} 0} \quad \text { for } R \leq 1-\frac{\Delta K_{\mathrm{th} / \mathrm{int}}}{\Delta K_{\mathrm{th} 0}}
$$




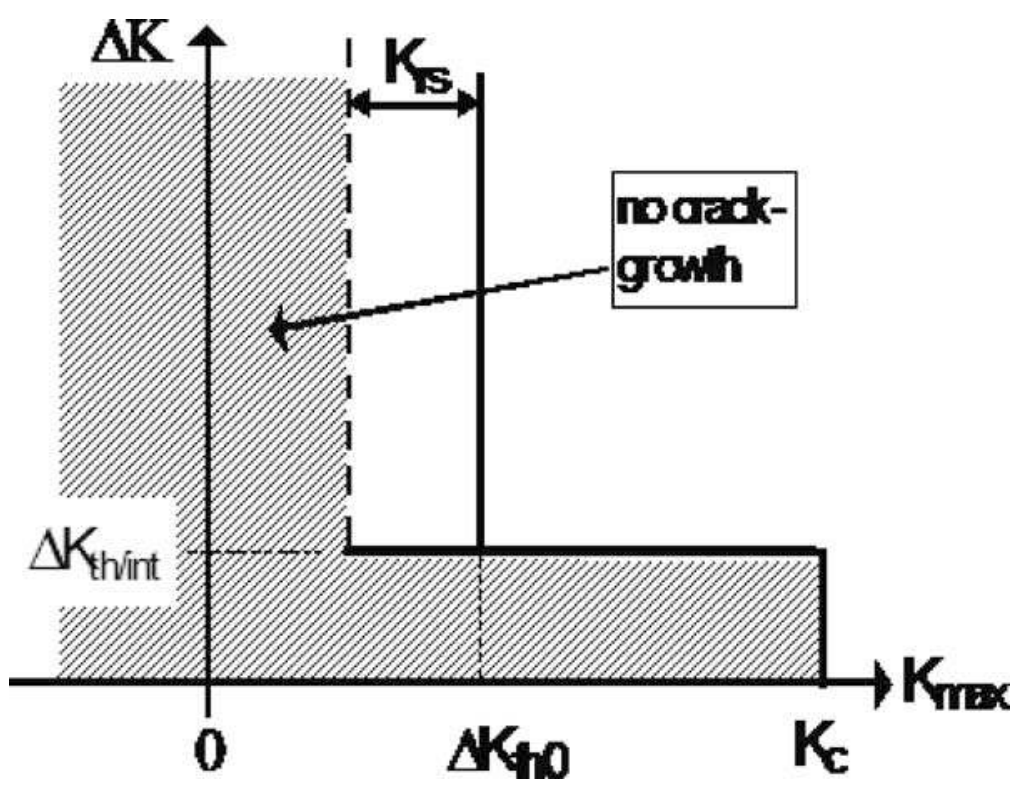

FIG. 6-Fatigue crack growth threshold represented in the $\Delta K$ versus $K_{\max }$ diagram.

$$
\Delta K_{I} \leq \Delta K_{\mathrm{th} / \mathrm{int}} \quad \text { for } R>1-\frac{\Delta K_{\mathrm{th} / \mathrm{int}}}{\Delta K_{\mathrm{th} 0}}
$$

The endurance criterion given by Eqs $13 \mathrm{a}$ and $13 \mathrm{~b}$ is represented by the L-shaped line in Fig. 6 . In the case of a crack emanating from a notch as considered here, $K_{\max }$ can be replaced by $K_{I N, \max }$ and $\Delta K_{I}$ by $\Delta K_{I N}$, which represent the maximum and the range of $K_{I N}$, respectively.

The SIF due to residual stresses should be either added to the left hand side of Eq 13a or subtracted from the right hand side. As mentioned in the Introduction, in brazed joints there are hardly any significant global residual stresses since the temperature history of the brazing process acts like a stress relief treatment. On the other hand, due to the mismatch of thermal expansion between the filler material and the bulk material, some local residual stresses are inevitable in the vicinity of the braze layer. Although the corresponding stress field acts only locally, they can give rise to a $\operatorname{SIF} K_{\text {Irs }}(a)$, which can affect fatigue crack growth, particularly in the threshold region. This is an inherent effect of the considered brazed seam, so it is suitable to include it on the right hand side of Eq 13a, which leads to

$$
\begin{gathered}
K_{I N, \max } \leq \Delta K_{\mathrm{th} 0}-K_{\mathrm{Nrs}} \quad \text { for } R \leq 1-\frac{\Delta K_{\mathrm{th} / \mathrm{int}}}{\Delta K_{\mathrm{th} 0}} \\
\Delta K_{I N} \leq \Delta K_{\mathrm{th} / \mathrm{int}} \quad \text { for } R>1-\frac{\Delta K_{\mathrm{th} / \mathrm{int}}}{\Delta K_{\mathrm{th} 0}}
\end{gathered}
$$

In Eq $14 \mathrm{a}, K_{\mathrm{Nrs}}$ denotes the local maximum of $K_{\mathrm{Irs}}(a)$ in the vicinity of the surface, i.e., for short crack lengths. This value can be obtained from the measured curves $K_{\text {Irs }}(a)$ that are obtained by the CC-method as described in the next section.

\section{Measurement of the Residual Stresses}

An efficient method to determine SIF due to residual stresses is the CC technique $[15,16]$. It enables the residual stress distribution on a complete cross-section as well as the resulting SIF to be measured. However, the disadvantage of the method is that it works only for two-dimensional components. Thus, structures in three dimensions like the considered impellers have to be cut in such a way that approximately plane slices are obtained. The corresponding stress-redistribution on the cross-section in question has to be measured and taken into account.

The brazed turbo compressor impellers shown in Fig. 1 was use to measure the residual stresses. The impeller consists of the soft martensitic stainless steel X3CrNiMo13-4. Brazing was performed in an 


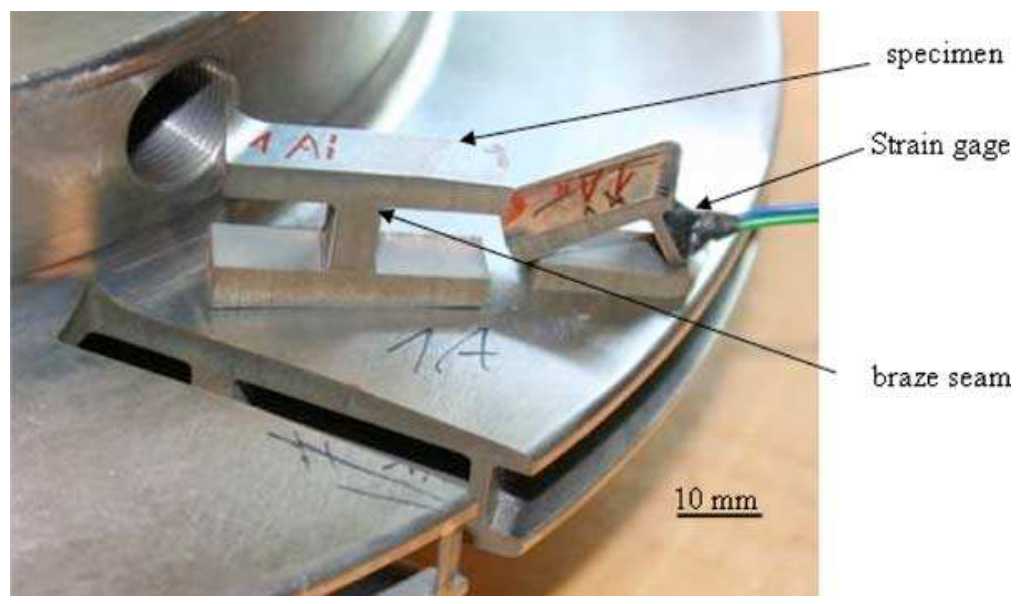

FIG. 7-H-shaped specimen (denoted by 1Ai) removed from the impeller by EDM-cutting.

industrial shielding gas furnace (SOLO Profitherm 600) at a temperature of $1020^{\circ} \mathrm{C}$ for 20 min. Hydroargon 7 (93 vol. \% Ar and 7 vol. \% $\mathrm{H}_{2}$ ) was used as shielding gas. The addition of hydrogen to the argon allows removing the oxide film on the stainless steel surface, which is essential for filler metal wetting. After brazing, the specimens were tempered at $520^{\circ} \mathrm{C}$ for $5.5 \mathrm{~h}$ in nitrogen atmosphere. A brazing zone width of approximately $100 \mu \mathrm{m}$ is finally achieved. To obtain specimens at representative locations a cut was introduced by electric discharge machining (EDM) in the impeller such that $\mathrm{H}$-shaped samples designated shown in Fig. 7 were obtained. The corresponding global stress release was measured by strain gage that can be seen on Fig. 7. As expected from the above discussion, no significant strains were measured by the strain gage due to cutting out the specimen. By a second cut, the H-shaped specimen shown in Fig. 7 was turned into a T-shaped one, on which the measurements described in the following were performed.

In order to apply the CC-method to a T-shaped specimen as small as the ones shown in Fig. 7, the procedure was modified such that the displacement $v$ could be measured (Fig. 8) instead of the normally measured back-face strain (see example in Ref 7). As derived in Ref 17 for the case of a measured strain, the SIF due to the residual stresses can be obtained analogously from a measured displacement such as $v(a)$ by the general relation

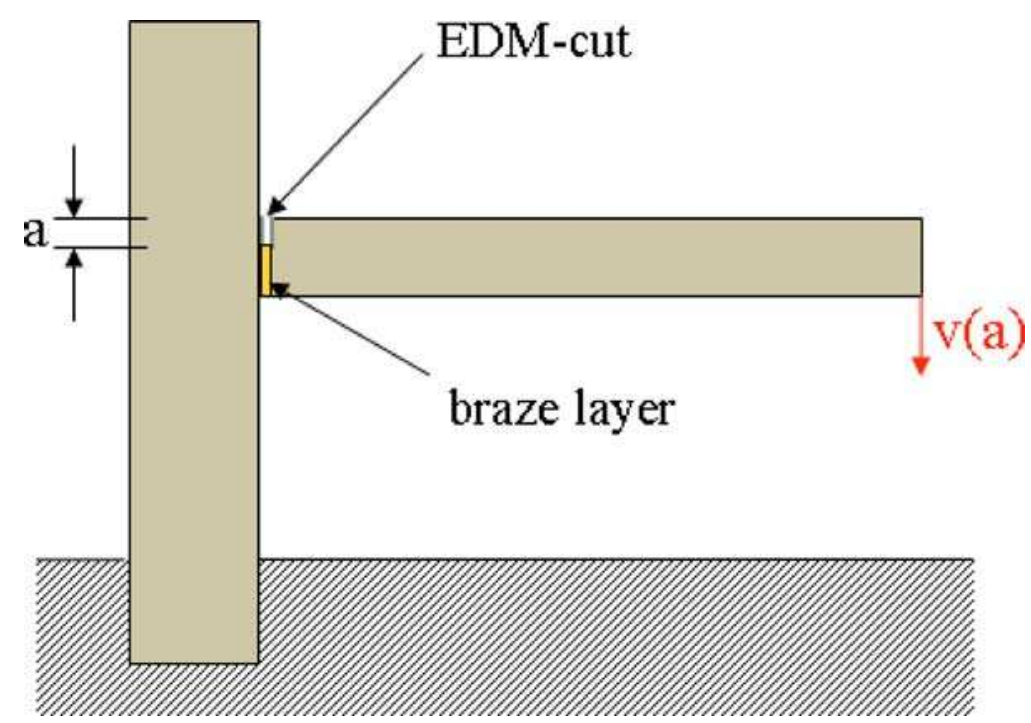

FIG. 8-Displacement $v$ at a T-shaped specimen due to cut of depth a along the brazed seam (schematically). 


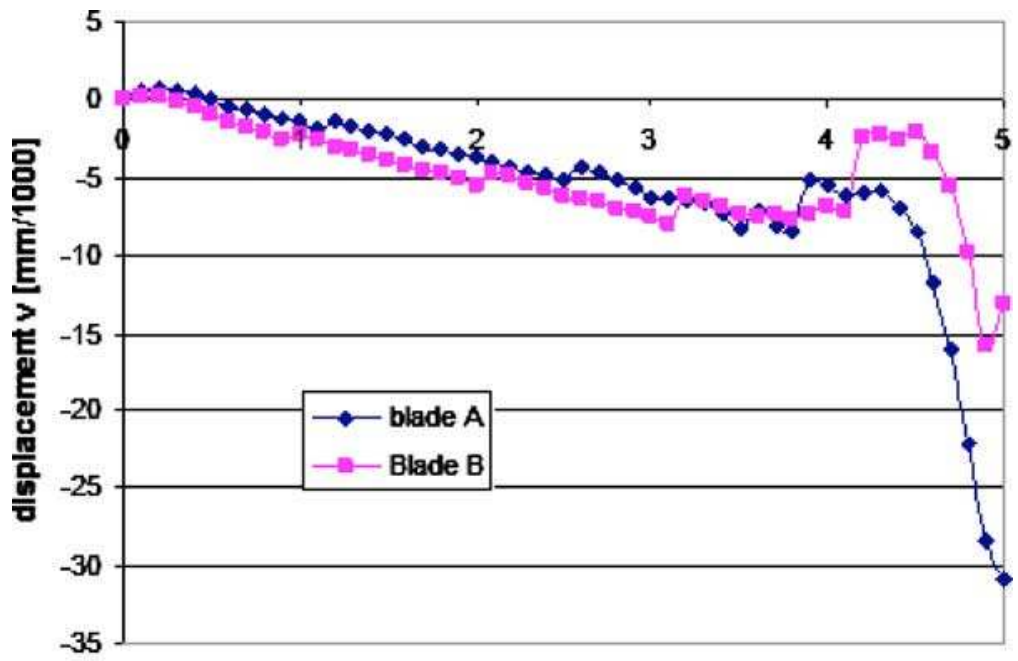

cut depth a [mm]

FIG. 9-Measured displacements at two arbitrary blades $A$ and $B$.

$$
K_{\mathrm{Irs}}(a)=\frac{E}{Z(a)} \cdot \frac{d v(a)}{d a}
$$

where:

$E=$ Young's modulus and

$Z(a)=$ corresponding influence function $[16,17]$.

Figure 9 shows the measured displacements. The $K_{\text {Irs }}(a)$ resulting from applying Eq 15 to the measured displacements are shown in Fig. 10. From the distribution of $K_{\mathrm{Irs}}(a)$, one can see that there is a very sharp tensile peak near the surface, followed by region of predominant compression. The dimples in the $K_{\text {Irs }}(a)$ curves are expected to reflect local inhomogeneities in the brazed layer such as clusters of voids. The agreement between blades A and B, which are two arbitrary blades from the same impeller, is relatively good, indicating that the measurements were repeatable.

\section{Fatigue Assessment of a T-Shaped Brazed Joint}

For the sake of simplicity we ignore the relatively small difference between the weighting factors of the bending and tensile stresses, respectively, that appear in Eqs 9a-9d, which simplify the latter to

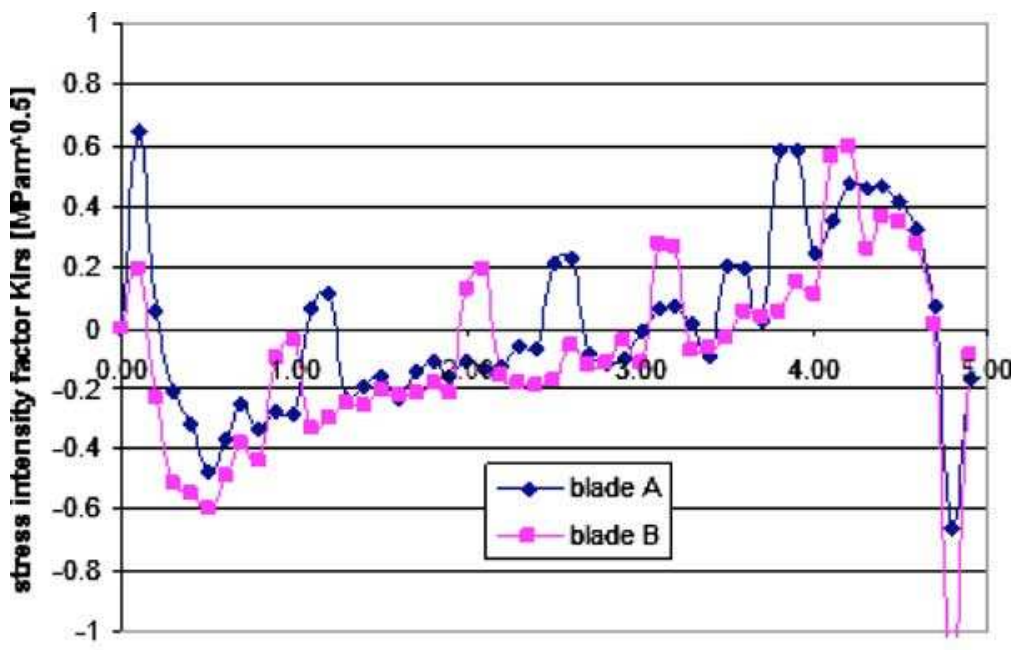

crack depth a [mm]

FIG. 10-SIF as a function of cut depth a obtained by Eq 15 from displacements shown in Fig. 9. 


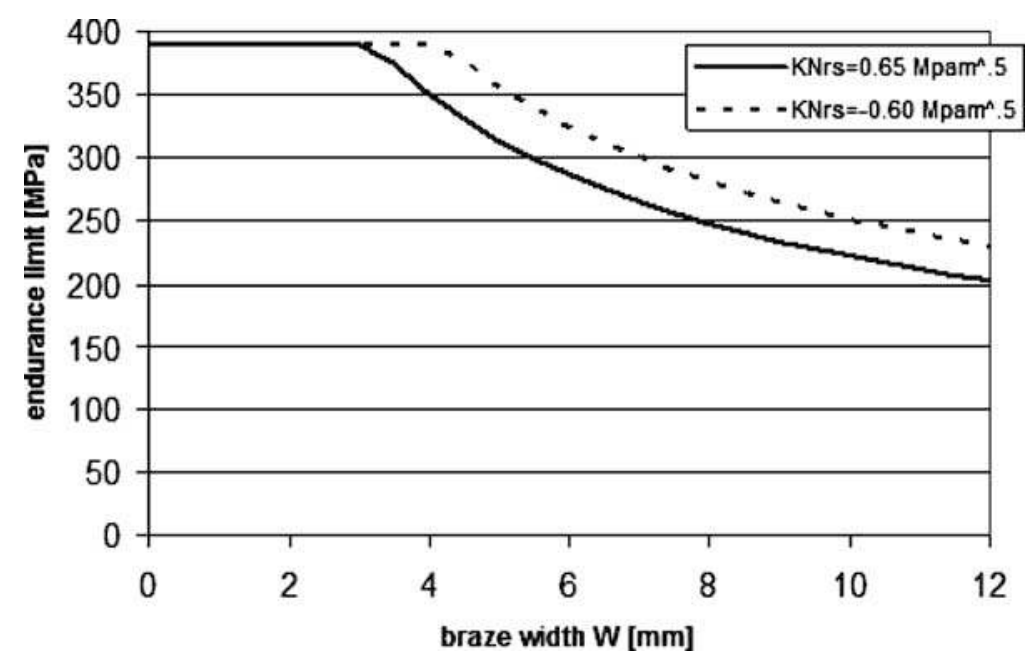

FIG. 11-Endurance limit (right hand side of Eq 18) for two cases of residual stress.

$$
K_{I N} \cong 0.422 \cdot \sigma_{\mathrm{app}} \cdot \sqrt{W}
$$

where:

$$
\sigma_{\text {app }}=\frac{6 M}{W^{2}}+\frac{N}{W}
$$

where:

$\sigma_{\text {app }}$ is the total nominal stress of the T-joint near the braze seam.

In the case of an impeller, $\sigma_{\text {app }}$ is the nominal stress perpendicular to the braze seam on the surface of a blade, which can be obtained by a finite element analysis.

By inserting Eqs 16 in 14a one obtains the following criterion for endurance:

$$
\sigma_{\mathrm{app}}<\frac{\Delta K_{\mathrm{th} 0}-K_{\mathrm{Nrs}}}{0.422 \cdot \sqrt{W}}
$$

The value of $\Delta K_{\mathrm{th} 0}$ corresponding to the lower bound of the measured $\Delta K_{\mathrm{th}}(R)$ (dotted line in Fig. 5) is $\Delta K_{\mathrm{th} 0}=10.0 \mathrm{MPa} \cdot \mathrm{m}^{0.5} . K_{\mathrm{Nrs}}$, which is defined as the maximum $K_{\mathrm{Irs}}$ in the range of relatively small cut depths $a$, is found from the measured curves in Fig. 10 to be roughly $K_{\mathrm{Nrs}}=0.65 \mathrm{MPa} \cdot \mathrm{m}^{0.5}$. However, the corresponding first peak is very narrow. After only a few tenths of a millimeter, $K_{\text {Irs }}$ becomes about $K_{\mathrm{Nrs}}=-0.60 \mathrm{MPa} \cdot \mathrm{m}^{0.5}$ and stays negative for a large range of crack depth. This effect is discussed below.

With these numerical values, the right hand side of Eq 18, which represents the theoretical endurance limit, is shown graphically in Fig. 11 as a function of the width of the braze seam, i.e., the thickness of the blade in the case of an impeller. In order to show the effect of the residual stresses, the less conservative case of $K_{\mathrm{Nrs}}=-0.60 \mathrm{MPa} \cdot \mathrm{m}^{0.5}$ is included for comparison. As $W$ approaches zero the endurance limit according to Eq 18 tends to infinity. Of course the curve is cut-off at the level of the endurance limit of the uniaxial tensile test, which is commonly assumed to be $\sim 0.4 \cdot R_{m}[13]$, with $R_{m} \approx 980 \mathrm{MPa}$ for the brazed joint considered here.

\section{Discussion and Conclusions}

The fatigue crack growth behavior of brazed joints is different from the one of bulk material. The threshold values are similar, but the subsequent $d a / d N$-curve rises much steeper with increasing $\Delta K$. Therefore it is not recommended to perform a crack growth calculation to show a safe-life behavior. There should be no growth at all of crack-like defects even for loads that occur only by relatively few cycles.

To show endurance for structures with stress concentrations and crack-like defects, an analysis based on fracture mechanics should be applied. A relatively simple method is suggested in this paper. Note that the initial crack size and shape do not appear explicitly in the corresponding equations. This is suitable since the defect size at the edge of a T-shaped braze seam is usually not known exactly. The endurance 
limit shown in Fig. 11 covers pre-existing cracks up to a few tenths of a millimeter. For known larger defect sizes, the full solution of $K_{I}(a)$ as given in Eqs $9 \mathrm{a}-9 \mathrm{~d}$ should be used instead of the NSIF $K_{I N}$, but it is expected that the presented simple solution covers most of the pre-existing defects. Unpublished experiments performed by one of the authors have revealed that typical brazing defects like voids as a result of incomplete gap filling exhibit a significantly higher resistance to growth than actual fatigue crack. This adds another component of safety.

Another interesting and important finding is that the derived endurance limit (Fig. 11) depends on the width $W$ of the braze seam, i.e., the width of the impeller blade in the present case. This means that thinner blades can withstand higher fatigue stresses than thicker ones.

From the few residual stress measurements performed so far, one can conclude that there are some residual stresses in the impellers, but they do not play a crucial role in the endurance limit. It seems to be clear that they only contribute to the $\Delta K_{\text {th }}$ by less than about $1.0 \mathrm{MPa} \cdot \mathrm{m}^{0.5}$ to the threshold, which means less than about $10 \%$. It is not quite clear yet whether they affect it in a positive or negative way: There is a very sharp positive peak of $K_{\text {Irs }}$ at a cut depth of about $0.1 \mathrm{~mm}$, followed by a large range of $a$, where $K_{\text {Irs }}$ is negative. Physically, the latter is expected to be more relevant since it increases the threshold in the long and crucial phase where the defect or crack size is longer than $0.1 \mathrm{~mm}$, whereas influencing factors in the initiation phase are less important. The effect of the residual stresses on the endurance limit is not very large anyway, as can be seen from the comparison of the two curves shown in Fig. 11. However, as long as this question is not resolved by further comparisons of experimental data, it is recommended to consider the more conservative value $K_{\mathrm{Nrs}}=0.65 \mathrm{MPa} \cdot \mathrm{m}^{0.5}$.

The procedure presented in this paper is a first attempt to establish a practical fatigue design concept for brazed structures. No considerations about adequate safety factors are made yet. The presented numbers and parameters have to be regarded as preliminary and informative. They need further justification by further experimental and theoretical data, particularly fatigue data from T-shaped brazed bonds, which are planned but not available yet.

\section{Acknowledgments}

The writers wish to thank MAN Turbo Switzerland, in particular Dr. Hans Gut, for enabling and supporting this work,

\section{References}

[1] Leinenbach, C., Schindler, H. J., Baser, T., Rüttimann, N., and Wegener, K., "Fracture Behaviour and Defect Assessment of Brazed Martensitic Stainless Steel Joints," Eng. Failure Anal., in press.

[2] Leinenbach, C., Schindler, H. J., Baser, T. A., Rüttimann, N., and Wegener, K., "Fracture Behaviour and Defect Assessment of Brazed Steel Joints," Proc. of the 17th European Conference on Fracture-ECF17, Brno, Czech Republic, September 5-9, 2008, pp. 2121-2128.

[3] Kassner, M. E., Kennedy, T. C., and Schrems, K. K., "The Mechanism of Ductile Fracture in Constrained Thin Silver Films," Acta Mater., Vol. 46, 1998, pp. 6445-6457.

[4] Tolle, M. C., Kassner, M. E., Cerri, E., and Rosen, R. S., "Mechanical Behaviour and Microstructure of Au-Ni Brazes," Metall. Mater. Trans. A, Vol. 26, 1995, pp. 941-8.

[5] Baser, T. A., Leinenbach, C., and Schindler, H. J., "Fracture Behaviour of Brazed Soft Martensitic Stainless Steel Joints Under Cyclic Loading," Mater. Sci. Forum, Vol. 636-637, 2010, pp. 14901495.

[6] Baser, T. A., Leinenbach, C., and Schindler, H. J., "Cyclic Fracture Behaviour of Brazed Steel Joints," Proc. of the 12th International Conference on Fracture (ICF12), Ottawa, July 12-17, 2009.

[7] Schindler, H. J., Sönnichsen, S., and Martens, H. J., "A Fracture Mechanics Approach to Estimate the Fatigue Endurance of Welded T-Joints Including Residual Stress Effects," Fatigue Fract. Eng. Mater. Struct., Vol. 30, 2006, pp. 206-213.

[8] Schindler, H. J., "Endurance Assessment for Notched Structural Parts with Imperfections," DVMBericht, Vol. 234, 2002, pp. 67-76 (in German).

[9] Schindler, H. J., "On the Significance of Crack Tip Shielding in Fatigue Threshold-Theoretical Relations and Experimental Implications," Fatigue Crack Growth Thresholds, Endurance Limits, and 
Design, ASTM STP 1372, J. C. Newman and R. S. Piascik, Eds., ASTM International, West Conshohocken, PA, 2000.

[10] Hertzberg, R. W., "On the Calculation of Closure-Free Fatigue Crack Propagation Data in Monolithic Metal Alloys," Mater. Sci. Eng., A, Vol. 190, 1995, pp. 25-32.

[11] Sadananda, K. and Shahinian, P., "Prediction of Threshold Stress Intensity for Fatigue Crack Growth using a Dislocation Model,” Int. J. Fract., Vol. 13, 1977, pp. 585-594.

[12] Riemelmoser, F. O. and Pippan, R., Fatigue Crack Growth Thresholds, Endurance Limits, and Design, ASTM STP 1372, J. C. Newman and R. S. Piascik, Eds., ASTM International, West Conshohocken, PA, 2000.

[13] Forschungskuratorium Maschinenbau, Stress Analysis Based on Fracture Mechanics, VDMA Verlag $\mathrm{GmbH}, 2001$, Frankfurt, Germany (in German).

[14] Han, S., Crack Growth Behaviour of Structural Steels in the Threshold Regime, Shaker Verlag, Aachen (Germany), 1996, Band 8/96 (in German).

[15] Prime, M. B., "Residual Stress Measurement by Successive Extension of a Slot: The Crack Compliance Method," Appl. Mech. Rev., Vol. 52, No. 2, 1999, pp. 75-96.

[16] Schindler, H. J., "Effects of Residual Stresses on Crack Growth and Structural Safety," Proceedings of the Ninth Int. Conf. on Mechanical Behaviour of Materials (ICM 9, Invited Sectional Lecture), Geneva, 2003.

[17] Schindler, H. J., Cheng, W., and Finnie, I., "Experimental Determination of Stress Intensity Factors Due to Residual Stresses,” Exp. Mech., Vol. 37, No. 3, 1997, pp. 272-277. 